CAVALCANTE, G.S. e SOUZA, F.S. Levantamento do uso de exame radiográfico na rotina de atendimentos em uma clínica veterinária na cidade de Manaus-AM. PUBVET, Londrina, V. 5, N. 37, Ed. 184, Art. 1244, 2011.

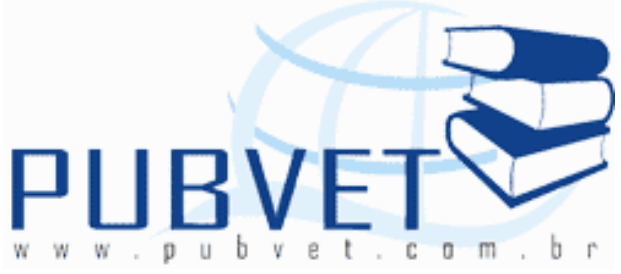

PUBVET, Publicações em Medicina Veterinária e Zootecnia.

\title{
Levantamento do uso de exame radiográfico na rotina de atendimentos em uma clínica veterinária na cidade de Manaus-AM
}

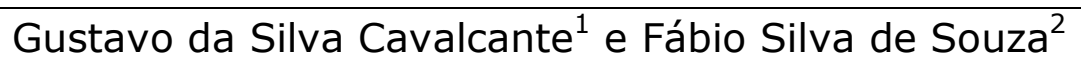

${ }^{1}$ Médico Veterinário autônomo. E-mail: gustavosultao@hotmail.com

${ }^{2}$ Prof. do curso de medicina veterinária da Escola Superior Batista do Amazonas. E-mail: souzamedvet@yahoo.com.br

\section{Resumo}

Os exames complementares por meio de imagens têm sido bastante empregados na medicina veterinária e se tornaram importantes no auxílio ao diagnóstico de diferentes patologias na clínica de pequenos animais permitindo ao veterinário estabelecer uma conduta de tratamento e um possível prognóstico dependendo do caso. O presente estudo teve por objetivos registrar o emprego de exame radiográfico na rotina de atendimentos em diferentes patologias; verificar a efetividade do exame radiográfico na abordagem do diagnóstico diferencial entre patologias e analisar a frequência de diferentes casos envolvendo o uso de exame radiográfico com os parâmetros de espécie animal, raça, sexo e idade que foram submetidos à avaliação radiográfica. O trabalho consistiu em um levantamento de casos oriundos de uma clínica veterinária na cidade de Manaus, sendo os dados obtidos a partir de fichas de avaliação clínica/cirúrgica de animais atendidos no 
CAVALCANTE, G.S. e SOUZA, F.S. Levantamento do uso de exame radiográfico na rotina de atendimentos em uma clínica veterinária na cidade de Manaus-AM. PUBVET, Londrina, V. 5, N. 37, Ed. 184, Art. 1244, 2011.

período de 19 de agosto a 20 de outubro de 2010. De 516 procedimentos realizados na rotina do local de estudo em 32 casos $(6,2 \%)$ houve a necessidade do emprego de exame radiográfico. De 32 casos analisados o exame radiográfico foi efetivo na condução do diagnóstico das patologias. Destes, $93,6 \%$ dos animais acometidos foram da espécie canina; $71 \%$ sendo animais de raça e destes $31,8 \%$ prevaleceu a raça Rottweiler; $64,5 \%$ foram machos e animais com idade de até dois anos $(51,6 \%)$ foram os que predominaram. O exame complementar por imagem radiográfica é uma importante ferramenta no auxílio diagnóstico na rotina de uma clínica veterinária.

Palavras-chave: Exame, radiodiagnóstico, patologias

\title{
Survey of the use of radiographs in the routine care in a veterinary clinic in the city of Manaus-AM
}

\begin{abstract}
The exams through images have been widely used in veterinary medicine and have become important for the diagnosis of different pathologies in the small animal clinic allowing the veterinarian to establish a pipeline to a treatment and possible prognosis depending on the case. This study aimed to record the use of radiographs in the routine care in different pathologies; verify the effectiveness of the radiographic examination in diagnosing the disease and analyze the differential between the frequency of different cases involving the use of X-ray with the parameters animal species, breed, sex and age who underwent radiographic evaluation. The work consisted of a survey of cases from a veterinary clinic in the city of Manaus, and the data obtained from clinical evaluation sheets/surgical animals treated between August 19 to October 20, 2010. Of 516 procedures performed in the study area 32 cases $(6.2 \%)$ was the need for the use of radiographic examination. In 31 cases
\end{abstract}


CAVALCANTE, G.S. e SOUZA, F.S. Levantamento do uso de exame radiográfico na rotina de atendimentos em uma clínica veterinária na cidade de Manaus-AM. PUBVET, Londrina, V. 5, N. 37, Ed. 184, Art. 1244, 2011.

analyzed the radiographic examination was effective in guiding the diagnosis of pathologies. Of these, $93.6 \%$ of affected animals were of the canine species, $71 \%$ being bred, and of these $31.8 \%$ prevailed Rottweiler, $64.5 \%$ were males and animals aged up to two years (51.6\%) were predominated. Diagnostic tests for radiographic image is an important tool in routine diagnostic aid in a veterinary clinic.

Keywords: Examination, radiology, pathology

\section{INTRODUÇÃO}

O diagnóstico por imagem constitui-se em um elemento de grande valor para os pacientes caninos e felinos que chegam à condição de emergência ou não à clínica veterinária. Na rotina veterinária, a radiografia é a principal modalidade de imagem na avaliação de afecções osteoarticulares e intratorácicas, além de fornecer uma visão panorâmica, informações sobre a cavidade abdominal (HULSE; JOHSON, 2002) e outras partes do corpo animal.

São várias as situações de patologias na rotina clínica veterinária que podem ser diagnosticadas por meio de exames complementares por diagnóstico por imagem, principalmente radiografias. Casos como de fraturas de diferentes tipos como as que envolvem ossos como rádio e ulna representam de $8,5 \%$ a $18 \%$ da casuística de fraturas nos cães e gatos, com a maioria dos autores relacionando incidência média de $17 \%$ constituindo-se o terceiro tipo mais frequente em cães (RUDD; WHITEHAIR, 1992; EGGER, 1993). As fraturas do fêmur também são algumas das mais comuns em medicina veterinária (HULSE; JOHSON, 2002). Assim como as fraturas de tíbia em cães e gatos correspondem a $20 \%$ de todos os ossos fraturados segundo Rudd e Whitehair (1992), tendo os acidentes automobilísticos como causa principal e ainda projeteis de armas de fogo, brigas entre cães, quedas e traumatismos de origem desconhecida como outras causas e geralmente estas 
CAVALCANTE, G.S. e SOUZA, F.S. Levantamento do uso de exame radiográfico na rotina de atendimentos em uma clínica veterinária na cidade de Manaus-AM. PUBVET, Londrina, V. 5, N. 37, Ed. 184, Art. 1244, 2011.

fraturas envolvem também a fíbula (SLATTER, 1998; BOJRAB, 2005; FOSSUM, 2005). Pode-se citar ainda, segundo Umphlet e Johnson (1990), Brinker (1999) e Gioso (2001), as fraturas de mandíbula e maxilar são geralmente causadas por traumas envolvendo atropelamentos, brigas, quedas ou ainda neoplasias ou doença periodontal. As luxações também fazem parte de traumas constatados na rotina de atendimentos veterinários sendo que as de patela seriam as mais comuns que acometem os joelhos dos cães (HULSE, 1981; TOMLISON; CONSTANTINESCEU, 1994). Esta afecção pode ser de origem congênita ou traumática, sendo a luxação de patela medial congênita a mais frequentemente observada (HAYES et al., 1994).

A displasia coxofemoral também é uma patologia diagnosticada por meio de exames radiográficos que pode estar associada a fatores nutricionais, biomecânicos e de meio ambiente ou à hereditariedade (SOMMER; FRATOCCHI 1998). Estudos mostram que $70 \%$ dos animais radiograficamente afetados não apresentaram sintomas e somente 30\% necessitaram de algum tipo de tratamento (GEROSA, 1995) e cerca de $80 \%$ dos cães displásicos mostram evidências radiológicas aos doze meses e em alguns casos, só são identificadas aos dois anos (LUST et al., 1985).

Tumores como, osteossarcomas, e presença de corpos estranhos no trato gastrointestinal tem ocorrido com uma certe frequência em atendimentos de rotina.

\section{METODOLOGIA}

Tratou-se de um estudo observacional de abordagem qualitativa, no qual realizou-se um levantamento de casos em uma clínica veterinária na cidade de Manaus. Os dados foram obtidos a partir de fichas de avaliação clínica/cirúrgica de animais atendidos em uma clínica veterinária no período de 19 de agosto a 20 de outubro de 2010 . Foram selecionados todos os casos que 
CAVALCANTE, G.S. e SOUZA, F.S. Levantamento do uso de exame radiográfico na rotina de atendimentos em uma clínica veterinária na cidade de Manaus-AM. PUBVET, Londrina, V. 5, N. 37, Ed. 184, Art. 1244, 2011.

envolveram a necessidade de complementação diagnóstica por meio de exames radiográficos para confirmação de patologias ou na realização de diagnóstico diferencial, em cães e gatos atendidos. Foram anotados dados referentes a características dos animais acometidos como: espécie, raça, sexo e idade. Os dados coletados foram acomodados no formato de tabelas e posteriormente analisados quanto à efetividade do exame radiográfico na abordagem do diagnóstico diferencial entre patologias e a analise da freqüência de diferentes casos envolvendo o uso deste tipo de exame complementar com os parâmetros de espécie animal, raça, sexo e idade dos animais.

\section{RESULTADOS E DISCUSSÃO}

No período de avaliação compreendido entre os dias 19 de agosto a 20 de outubro de 2010 foram registrados 516 procedimentos nestes três meses. $\mathrm{Na}$ Tabela 1 está demonstrado na forma de categorias, as ocorrências de atendimento durante o período de avaliação e o total de cada tipo das mesmas.

Tabela 1. Discriminação por categoria do número total de procedimentos realizados no período de agosto a outubro de 2010 em uma clínica veterinária na cidade de Manaus-AM.

\begin{tabular}{lccccc}
\hline \multirow{2}{*}{ Categorias } & \multicolumn{3}{c}{ Meses } & \multirow{2}{*}{ Total } \\
\cline { 2 - 5 } & Agosto & Setembro & Outubro & \\
\hline Consultas & 64 & 86 & 66 & 216 \\
Vacinações & 41 & 60 & 49 & 150 \\
Fluidoterapia e aplicação de medicamentos & 18 & 15 & 20 & 53 \\
Cirurgias diversas & 18 & 10 & 15 & 43 \\
Cirurgias ortopédicas & 5 & 7 & 10 & 22 \\
Raio-X & 5 & 7 & 20 & 32 \\
\hline Total & 151 & 185 & 180 & 516 \\
\hline
\end{tabular}


CAVALCANTE, G.S. e SOUZA, F.S. Levantamento do uso de exame radiográfico na rotina de atendimentos em uma clínica veterinária na cidade de Manaus-AM. PUBVET, Londrina, V. 5, N. 37, Ed. 184, Art. 1244, 2011.

Inferiu-se que do total de atendimentos realizados, 6,2\% (32) dos casos necessitaram do uso de raio-x como exame complementar. Nos 32 casos obtidos em que houve a solicitação de raio-x as suspeitas levantadas para tal foram diferentes e estão demonstradas na Tabela 2.

Tabela 2. Discriminação das suspeitas envolvidas na solicitação de raio-x como diagnóstico complementar em 32 casos atendidos no período de agosto a outubro de 2010 em uma clínica veterinária na cidade de Manaus-AM.

\begin{tabular}{lcccc}
\hline \multicolumn{1}{c}{ Categorias } & \multicolumn{3}{c}{ Meses } & \multirow{2}{*}{ Total } \\
\cline { 2 - 5 } & Agosto & Setembro & Outubro & \\
\hline Fraturas & 5 & 7 & 10 & 22 \\
Displasia Coxofemoral & & & 4 & 4 \\
Luxação & & & 2 & 2 \\
Osteossarcoma & & & 2 & 2 \\
Corpo estranho & & & 1 & 1 \\
'Outro' - Suspeita de tiro & 5 & 7 & 20 & 32 \\
\hline Total & & & & \\
\hline
\end{tabular}

Os resultados demonstram um crescente aumento no número de casos que necessitaram do apoio de raio-x como exame complementar. Em agosto contabilizou-se cinco casos apenas contrapondo o mês de outubro onde se observou um total de 20 casos envolvendo radiodiagnóstico.

Evidencia-se um número expressivo de $22(68,7 \%)$ casos envolvendo fraturas, relativo ao total de exames radiológicos e uma freqüência baixa de atendimentos envolvendo corpos estranhos (um caso, $[3,1 \%]$ ) e 'outros', atendido como suspeita de projétil por arma de fogo também de um caso $(3,1 \%)$.

Em uma situação de casos envolvendo números ainda baixos, comparando-se com o total de exames radiográficos observa-se, quatro ocorrências $(12,5 \%)$ de displasia coxofemoral, duas $(6,3 \%)$ de luxação e duas $(6,3 \%)$ de osteossarcoma (Tabela 2$)$.

O exame complementar por meio do diagnóstico por imagem envolvendo raio-x foi importante na confirmação das suspeitas clínicas e na confirmação do 
CAVALCANTE, G.S. e SOUZA, F.S. Levantamento do uso de exame radiográfico na rotina de atendimentos em uma clínica veterinária na cidade de Manaus-AM. PUBVET, Londrina, V. 5, N. 37, Ed. 184, Art. 1244, 2011.

diagnóstico como demonstrado a seguir na Tabela 3 envolvendo 21 dos 22 casos de fraturas registrados segundo a raça, sexo, idade, localização da fratura e a respectiva causa. Ressalta-se que um caso envolvendo fratura foi diagnosticado, porém o proprietário desistiu de levar adiante a avaliação no local.

Tabela 3. Discriminação de 21 casos registrados de fraturas envolvidas na solicitação de raio-x como diagnóstico complementar, segundo, espécie, raça, sexo, idade, localização da fratura e a causa, no período de agosto a outubro de 2010 em uma clínica veterinária na cidade de Manaus-AM.

\begin{tabular}{|c|c|c|c|c|c|c|}
\hline $\begin{array}{c}\text { Nome } \\
\text { do } \\
\text { animal }\end{array}$ & Espécie & Raça & Sexo & Idade & $\begin{array}{l}\text { Localização } \\
\text { da fratura }\end{array}$ & Causa \\
\hline $\mathrm{S} / \mathrm{N}$ & Ave & - & - & - & Fêmur & Atropelamento \\
\hline Pepa & Felina & Siamês & $M$ & 6 meses & Pelve & Atropelamento \\
\hline Barão & Canina & Rottweiler & $M$ & 3 anos & Úmero & Briga \\
\hline Maike & Canina & American & $M$ & 12 anos & Úmero & Brincando em casa \\
\hline Simba & Canina & $\begin{array}{l}\text { Pastor } \\
\text { Alemão }\end{array}$ & M & 2 anos & Úmero & Briga \\
\hline Rot & Canina & Rottweiler & $M$ & 2 anos & Úmero & Atropelamento \\
\hline Street & Canina & Poodle & $M$ & 2 anos & Fêmur & Atropelamento \\
\hline Dimy & Canina & Pinscher & $M$ & 2 anos & Fêmur & Caiu do colo \\
\hline Luck & Canina & SRD & $M$ & 2 anos & Fêmur & Atropelamento \\
\hline Acamp & Canina & SRD & $M$ & 2 anos & Radio e Ulna & Caiu da janela \\
\hline Zeus & Canina & Chow-chow & M & 4 anos & Radio e Ulna & Briga \\
\hline Toto & Canina & SRD & $M$ & - & Tíbia & Atropelamento \\
\hline Tyson & Canina & SRD & $M$ & 4 meses & Tíbia & Atropelamento \\
\hline Era & Canina & Rottweiler & $\mathrm{F}$ & 4 anos & - & Briga \\
\hline Jully & Canina & York & $\mathrm{F}$ & 4 meses & Mandíbula & Atropelamento \\
\hline Capitu & Canina & Rottweiler & $\mathrm{F}$ & 3 anos & Mandíbula & Briga \\
\hline Amora & Canina & Poodle & $\mathrm{F}$ & 3 anos & $\begin{array}{c}\text { As duas Radio } \\
\text { e Ulna }\end{array}$ & Atropelamento \\
\hline Maggie & Canina & Pinscher & $\mathrm{F}$ & 2 anos & Radio e Ulna & Caiu da cama \\
\hline Maria & Canina & SRD & $\mathrm{F}$ & 6 anos & Radio e Ulna & Atropelamento \\
\hline Belinha & Canina & SRD & $\mathrm{F}$ & 4 meses & Fêmur & Atropelamento \\
\hline Bolinha & Canina & SRD & $\mathrm{F}$ & 5 anos & Fêmur & Atropelamento \\
\hline
\end{tabular}

Analisando-se apenas o perfil dos indivíduos atendidos cuja queixa clínica foi fratura, o que foi constatado após a tabulação dos dados está demonstrado na sequência: 
CAVALCANTE, G.S. e SOUZA, F.S. Levantamento do uso de exame radiográfico na rotina de atendimentos em uma clínica veterinária na cidade de Manaus-AM. PUBVET, Londrina, V. 5, N. 37, Ed. 184, Art. 1244, 2011.

Foi verificado que $90,4 \%$ (19) dos animais atendidos pertenceram a espécie canina e 4,8\% (um) pertenceu a felina e 4,8\% (um indivíduo) tratouse de uma ave.

Quando o aspecto analisado foi a raça $61,9 \%$ (13) dos animais atendidos tinham uma raça definida enquanto $38,1 \%$ (sete indivíduos) eram sem raça definida.

Quanto ao sexo dos animais acometidos verificamos um maior percentual de machos, 57,1\% (12 indivíduos) quando comparado ao de fêmeas que foi de $38,1 \%$ (oito) fêmeas além de uma ave $(4,8 \%)$ que não foi realizado a sexagem da mesma.

O perfil de idade dos animais atendidos foi de sua maioria jovens (até dois anos de idade) com percentual de 52,4\% (11 animais); de animais adultos ( $>2$ e <8anos) foi de $33,3 \%$ (sete animais) e de animais senis ( $>8$ anos) sendo de $4,8 \%$ (dois animais).

Os dados obtidos (Tabela 3) concordam com a abordagem realizada por Kealy e McAllister (2005) ao mencionar que das alterações que ocorrem em maior freqüência, quando o assunto é o sistema ósseo, estariam envolvidas as afecções traumáticas. Pois dos 31 exames realizados 21 foram de fraturas e de diferentes tipos onde foi observado um maior percentual de atendimentos a indivíduos com fratura de fêmur $28,6 \%$ correspondendo a seis casos sendo talvez umas das mais comumente observadas; seguidas por fraturas envolvendo rádio e ulna com $23,8 \%$ (cinco casos) que segundo alguns autores (RUDD; WHITEHAIR, 1992; EGGER, 1993) este tipo de fraturas representariam $8,5 \%$ a $18 \%$ da casuística de fraturas em cães e gatos constituindo-se o terceiro tipo mais freqüente em cães com uma incidência média de $17 \%$; as fraturas de úmero corresponderam a 19\% (quatro casos); já as fraturas de tíbia, segundo colocam Rudd e Whitehair (1992), corresponderiam a $20 \%$ de todos os ossos fraturados de animais atendidos, no entanto, na presente avaliação, foi constatado apenas dois casos $(9,5 \%)$ e ainda foram registrados 
CAVALCANTE, G.S. e SOUZA, F.S. Levantamento do uso de exame radiográfico na rotina de atendimentos em uma clínica veterinária na cidade de Manaus-AM. PUBVET, Londrina, V. 5, N. 37, Ed. 184, Art. 1244, 2011.

dois casos $(9,5 \%)$ com fratura de mandíbula, além de uma fratura de pelve $(4,8 \%)$ e um $(4,8 \%)$ caso que não foi identificado o tipo de fratura.

$\mathrm{Na}$ avaliação das causas desses 21 atendimentos envolvendo fraturas (Tabela 3) 57,1\% (12 casos) das ocorrências foram motivadas por atropelamentos; brigas corresponderam a 23,8\% (cinco casos); quedas também tiveram um índice elevado com 14,3\% (três casos) e por brincadeiras na residência registrou-se um caso apenas (4,8\%). Atropelamento é uma das causas mais apontadas por diferentes autores quando se trata de fraturas, representados principalmente, por acidentes automobilísticos, além de outros pequenos traumas como saltos ou quedas também seriam as causas mais comuns e Schwarz e Schrader (1984) reforçam este fato ao registrarem que de 16 cães estudados por eles 15 sofreram trauma por atropelamento e 11 de 12 gatos caíram de apartamentos. Atropelamentos ou quedas são os grandes responsáveis por fraturas tanto de fêmur como de rádio e ulna incluindo grande percentagem de fraturas de tíbia principalmente por atropelamento como foi registrado aqui (Tabela 3), porém tratando-se deste tipo de osso outras causas têm sido relatadas como projeteis de armas de fogo, brigas entre cães e traumatismos de origem desconhecida (SLATTER, 1998; FOSSUM, 2005).

As fraturas de mandíbula relatadas no presente levantamento, também envolveram atropelamento e brigas o que concorda com Umphlet e Johnson (1990), Brinker (1999) e Gioso (2001) que colocaram atropelamentos, brigas, quedas ou ainda neoplasias ou doença periodontal, como possíveis causas deste tipo de traumatismo.

Quando se avaliou os outros tipos de diagnóstico ocorridos levando-se em consideração as também características como raça, sexo, idade e ocorrência (Tabela 4), foi verificado que do total de atendimentos, em número de dez animais, $90 \%$ (nove) foram animais de raça definida enquanto $10 \%$ (um) não tinham definição da mesma. Em relação ao sexo $80 \%$ (oito 
CAVALCANTE, G.S. e SOUZA, F.S. Levantamento do uso de exame radiográfico na rotina de atendimentos em uma clínica veterinária na cidade de Manaus-AM. PUBVET, Londrina, V. 5, N. 37, Ed. 184, Art. 1244, 2011.

indivíduos) foram machos e $20 \%$ (dois indivíduos) de fêmeas apenas. Os animais classificados como jovens (até dois anos de idade) corresponderam a $50 \%$ (cinco animais) do total enquanto 30\% (três animais) eram animais adultos e apenas $20 \%$ (dois animais) eram senis (>8anos).

Das patologias registradas, $40 \%$ (quatro animais) corresponderam a displasia coxofemoral; $20 \%$ (dois indivíduos) foi por luxação e osteossarcoma cada um; um indivíduo (10\%) diagnosticado com corpo estranho e um (10\%) por outra causa diferente das anteriores.

A displasia coxofemoral tem-se destacado como uma das patologias mais estudadas na medicina veterinária segundo Makie et al. (2000) e coincidiu como a de maior ocorrência no período estudado. Segundo Lust et al. (1985), cerca de $80 \%$ dos cães displásicos mostram evidências radiológicas aos doze meses e, em alguns casos, só são identificadas aos dois anos. Porém dos quatro animais atendidos na rotina da clínica veterinária em Manaus-AM, a idade que prevaleceu correspondeu a de oito anos em três dos quatro animais atendidos, esta identificação tardia pode ser justificada, como mencionado por Gerosa (1995), que em alguns estudos foi mostrado que $70 \%$ dos animais radiograficamente afetados não apresentavam sintomas e somente $30 \%$ necessitam de algum tipo de tratamento.

Casos de luxação também estiveram presentes, principalmente a de patela, marcando sua presença, pois ocorreu em um dos indivíduos com idade de dois anos tratando-se de uma patologia comumente encontrada acometendo cães (HULSE, 1981; TOMLINSON; CONSTANTINESCEU, 1994), sendo esta afecção congênita ou traumática.

Outra ocorrência de relevância foi a de osteossarcomas em dois animais um com nove e outro com doze anos sendo o primeiro da raça Pastor Alemão e o segundo um animal sem raça definida. Este fato concorda com a colocação feita por Lacreta (2002), de que os osteossarcomas ocorrem, principalmente, em cães de meia idade e em raças de grande porte ou gigante e representam 
CAVALCANTE, G.S. e SOUZA, F.S. Levantamento do uso de exame radiográfico na rotina de atendimentos em uma clínica veterinária na cidade de Manaus-AM. PUBVET, Londrina, V. 5, N. 37, Ed. 184, Art. 1244, 2011.

cerca de $80 \%$ a $95 \%$ das neoplasias ósseas diagnosticadas em cães (JONGEWARD, 1985; BERG et al., 1990; STIMSON et al., 2000). Tanto cães machos quanto fêmeas podem ser acometidos por osteossarcomas (SPODNICK et al., 1992; SILVEIRA, 1996). Embora os resultados apresentados tenham sido de $100 \%$ machos atendidos estes achados não consistem entre todas as publicações (OLIVEIRA; SILVEIRA, 2008). Sabendo-se que é uma patologia com opções de tratamentos limitados, como colocado por Silveira (1996), os mesmos descrevem que os tratamentos realizados não alteram o resultado fatal da doença, apenas aumentam a sobrevida do animal. A amputação do membro ainda é o tratamento tradicional de cães com osteossarcoma apendicular e se tornou a medida adotada em ambos os casos apresentados. Entretanto, o uso de antiinflamatórios não esteroidais pode ser empregado para aliviar a dor em curto prazo, diminuindo a tensão e desconforto (MEDEIROS et al., 2010).

Também foi observado um caso de corpo estranho. Casos de corpos estranhos podem estar ligados a acidentes condicionados ao comportamento apresentado pelo animal como comer com avidez e engolir a comida sem mastigar, gostar de dilacerar tudo o que levam à boca e do costume mexer nos sacos de lixo quando tem a oportunidade (BURROWS et al., 1997). A avaliação radiográfica demonstra ser uma ótima avaliação complementar e pode ser a primeira modalidade de imagem utilizada em casos de suspeita de anormalidade gastrintestinal motivada por corpos estranhos, devido a capacidade de reconhecê-los radiograficamente sendo muito útil, principalmente, quando outros sinais de obstrução estiverem ausentes (LAMB, 1999). 
CAVALCANTE, G.S. e SOUZA, F.S. Levantamento do uso de exame radiográfico na rotina de atendimentos em uma clínica veterinária na cidade de Manaus-AM. PUBVET, Londrina, V. 5, N. 37, Ed. 184, Art. 1244, 2011.

Tabela 4. Discriminação de 10 casos registrados envolvendo diferentes diagnósticos por meio da solicitação de raio-x, segundo a raça, sexo, idade, no período de agosto a outubro de 2010 em uma clínica veterinária na cidade de Manaus-AM.

\begin{tabular}{ccccc}
\hline $\begin{array}{c}\text { Nome do } \\
\text { animal }\end{array}$ & Raça & Sexo & Idade & Diagnóstico \\
\hline Tom & Fila Brasileiro & M & 5 meses & Luxação \\
Maya & Pinscher & $\mathrm{F}$ & 2 anos & Luxação Patelar \\
Sara & Rottweiler & $\mathrm{F}$ & 8 anos & DCF \\
Rock & Pastor alemão & $\mathrm{M}$ & 8 anos & DCF \\
Sadan & Pit Bull & $\mathrm{M}$ & 1 ano e 2 & DCF \\
Negão & Rottweiler & $\mathrm{M}$ & meses & DCF \\
Gigante & Pinscher & $\mathrm{M}$ & 2 anos & Suspeita de tiro \\
Brutos & Rottweiler & $\mathrm{M}$ & 4 meses & Corpo estranho \\
Bruto & Pastor Alemão & $\mathrm{M}$ & 9 anos & Osteossarcoma \\
Bob & SRD & $\mathrm{M}$ & 12 anos & Osteossarcoma \\
\hline DCF=Displasia coxofemoral & & &
\end{tabular}

\section{CONCLUSÕES}

Com base nos objetivos propostos pode-se concluir que:

- $\quad$ De 516 procedimentos realizados na rotina do local de estudo em 32 casos $(6,2 \%)$ casos houve a necessidade do emprego de exame radiográfico;

- De 31 casos analisados o exame radiográfico foi efetivo na condução do diagnóstico das patologias;

- De 31 casos analisados, 93,6\% dos animais acometidos foram da espécie canina; $71 \%$ sendo animais de raça e destes $31,8 \%$ prevaleceu a raça Rottweiler; $64,5 \%$ foram machos e a animais com idade de até dois anos $(51,6 \%)$ foram os que predominaram.

\section{REFERÊNCIAS BIBLIOGRÁFICAS}

BERG, J.; LAMB, C. R.; O'CALLAGHAN, M. W. Bone scintigraphy in the initial evaluation of dogs with primary bone tumors. Journal of Americam Veterinary Assocition, v. 196, n. 6, 1990. BOJRAB, M. J. Técnicas atuais em cirurgia de pequenos animais. 3 . ed. São Paulo: Editora Roca, 2005. 425 p. 
BRINKER, P. D. L.; FLO, R. L. Ortopedia e tratamento das fraturas dos pequenos animais. São Paulo: Manole, 1999.

BURROWS, C. F.; BATT, R. M.; SHERDING, R. G. Afecções do intestino delgado. In: ETTINGER, S. J.; FELDMAN, E. C. Tratado de medicina interna veterinária. São Paulo: Editora Manole. 1997. p. $1618-1705$.

EGGER, E. L. Skull and mandibular fractures. In: SLATTER, D. Textbook of small animal surgery. 2. ed. Philadelphia: Editora Saunders, 1993, v. 2, cap. 142, p. 1910-1920.

FOSSUM, T. W. Cirurgia de pequenos animais. 2. ed. São Paulo: Editora Roca, 2005. 1390 p.

GEROSA, R. M. La displasia de la cadera canina desde una visión biomecánica.Revista de Medicina Veterinária, v. 76, n. 1, p. 69-71, 1995.

GIOSO, M. A. Análise clínica e histológica da utilização da resina acrílica autopolimerizável nas fraturas de mandíbula e maxila e separação da sínfese mentoniana em cães e gatos. Ciência Rural, v. 31, n. 2, p. 291-298, 2001.

HAYES, A. G.; BOUDRIEAU, R. J.; HUNGERFORD, L. L. Frequency and distribution of medial and lateral patellar luxation in dogs: 124 cases (1982-1992). Journal American Veterinary Medicine Association, v. 205, p. 716-720, 1994.

HULSE, D. A. Pathophysiology and management of medial patellar luxation in the dog. Veterinary Medicine Small Animal Clinical, v. 76, p. 43-51, 1981.

HULSE, D. A.; JOHSON, A. L. Fundamentos da Cirurgia Ortopédica e tratamento de Fraturas. In: Cirurgia de Pequenos Animais, $1^{\text {a }}$ ed., São Paulo, Roca, 2002, p. 787-853.

JONGEWARD, S. J. Primary bone tumors. Veterinary Clinics North America: Small Animal Practice, v. 15, n. 3, p. 609-637, 1985.

KEALY, J. K.; MCALLISTER, H. Ossos e Articulações. In: Radiologia e ultrassonografia do Cão e Gato. São Paulo: Editora Manole, 2005. p. 253-297.

LACRETA, J. Osteossarcoma pélvico em um cão da raça Rottweiler - relato de caso. In: XXIX. Congresso Brasileiro de Medicina Veterinária, Gramado. Anais em Cd room, 2002.

LAMB, C. R. Recent developments in diagnostic imaging of the gastrointestinal tract of the dog and cat. Veterinary Clinical of North America Small Animals Practice, v. 29, n. 2, p. 307-342, 1999.

LUST, G.; RENDANO, U. T.; SUMMERS, B. A. Canine hip dysplasia: concepts and diagnosis. Journal of the American Veterinary Medical Association, v. 187, p. 638-640, 1985.

MAKIE, K.; LINAMO, A.E.; OJALA, M. Estimates of genetic parameters for hip and elbow dysplasia in Finnish Rottweilers. Journal Animal Science, v. 78, n.5, p. 1141-1148. 2000.

MEDEIROS, F.; ROCHA, J. R.; SANTOS, M. S. P.; FRIOLANI, M. Osteossarcoma em cães Relato de caso. Revista Científica Eletrônica de Medicina Veterinária, n. 14. p. 5, 2010.

OLIVEIRA, F.; SILVEIRA, P. R. Osteossarcoma em cães (Revisão de literatura). Revista Científica Eletrônica de Medicina Veterinária, n. 11, 2008. 
RUDD, R. G.; WHITEHAIR, J. G. Fractures of the radius and ulna. Veterinary Clinical North American Small Animal Practice, v. 22, n. 1, p. 135-148, 1992.

SCHWARZ, P. D.; SCHRADER, S. C. Ulnar fracture and dislocation of the proximal radial epiphysis (Monteggia lesion) in the dog and cat: a review of 28 cases. Journal of the American Veterinary Medical Association, v. 185, n. 2, p. 190-194, 1984.

SILVEIRA, P. R. Estudo retrospectivo de osteossarcoma apendicular em cães, no período de janeiro de 2001 à janeiro de 2004. Estudo. Dissertação (Mestrado), Universidade Estadual Paulista. Faculdade de Ciências Agrárias e Veterinárias, Campus de Jaboticabal, 1996.

SLATTER, D. Manual de cirurgia de pequenos animais. 2. ed. São Paulo: Editora Manole, 1998. v. 1, p. 427-428.

SOMMER, E. L.; FRATOCCHI, C. L. G. Displasia Coxofemoral. Revista de Educação Continuada do CRMV-SP, v. 1, n. 1, p. 031-035, 1998.

SPODNICK, G.J.; BERG, J.; RAND, W.M. Prognosis for dogs with appendicular osteosarcoma treated by amputation alone: 162 cases (1978-1988). Jounal of the American Veterinary Association, v. 200, n. 7 p. 995-999, 1992.

STIMSON, E. L.; COOK, W. T.; SMITH, M. M.; FORRESTER, S. D.; MOON, M. L.; SAUNDERS, G. K. Extraskeletal osteosarcoma in the duodenum of cat. Journal Americam Animimal Hospital Association, v. 36, n. 4, p. 332-6, 2000.

TOMLINSON, J.; CONSTANTINESCU, G. M. Repair of medial patellar luxation. Veterinary Meicine, v. 89, p. 48-56, 1994.

UMPHLET, R. C.; JOHNSON, A. L. Mandibular fractures in the dog: a retrospective study of 157 cases. Veterinary Surgery, v. 19, p. 272, 1990. 